DOI: $10.17957 / \mathrm{IJAB} / 15.1758$

http://www.fspublishers.org

\title{
Pathogenic Microorganisms Infecting Berries in Mexico
}

\author{
Edith Garay-Serrano ${ }^{1,2 *}$, Samuel Cruz-Esteban ${ }^{1,2}$, Sylvia P. Fernández Pavia ${ }^{3}$, Gerardo Rodríguez Alvarado ${ }^{3}$ and \\ Nuria Gómez-Dorantes ${ }^{3}$ \\ ${ }^{1}$ Instituto de Ecología, A.C. Red de Diversidad Biológica del Occidente Mexicano. Avenida Lázaro Cárdenas 253, 61600 \\ Pátzcuaro, Michoacán, México \\ ${ }^{2}$ CONACYT. Avenida Insurgentes Sur 1582, 03940 Ciudad de México, México \\ ${ }^{3}$ Instituto de Investigaciones Agropecuarias y Forestales, Universidad Michoacana de San Nicolás de Hidalgo, Unidad Posta \\ Zootecnica, Carretera Morelia, Zinapécuaro km 9.5, C.P. 58880, Tarímbaro, Michoacán, México \\ "For correspondence: edith.garay@inecol.mx \\ Received 04 September 2020; Accepted 25 January 2021; Published 16 April 2021
}

\begin{abstract}
Mexico is one of the major producers of berries worldwide and ranks third amongst the principal exporters of these fruits. However, the presence of diseases in crops of blueberry, blackberry, strawberry, and raspberry in production areas of the country, has a negative impact in production yields. In this work, we presented a revision of all pathogens reported for these berries during the last 64 years in Mexico. Data on the different groups of pathogens including bacteria, stramenopila, and fungi, as well as location and references, are listed for the several types of berries produced. The pathogen species names were actualized according to the current taxonomic status following the specialized nomenclatural websites. Perspectives for future research are discussed. (C) 2021 Friends Science Publishers
\end{abstract}

Keywords: Blackberry; Blueberry; Oomycota; Phytopathogenic bacteria; Raspberry; Strawberry

\section{Introduction}

Berries are becoming some of the favorite fruits in many countries. During 2018, the total global production of berries was 6.16 million ton (FAOSTAT 2020). The United States, Mexico and Poland accounted for $54 \%$ of the total production worldwide in 2018. Mexico was the world's leading producer of blackberries (Rubus ulmifolius) with 287,125 tons, second producer of raspberries (Rubus idaeus) with 130,187 tons, the second in strawberry (Fragaria $\mathrm{x}$ ananassa) production with 653,639 tons, and the fifth producer of blueberries (Vaccinium corymbosum) with 40,251 tons (FAOSTAT 2020).

In Mexico, berries are produced all year round using irrigated and non-irrigated systems (SIAP 2018; SADERSIAP 2019). Among the 23 states that cultivate berries (Fig. 1 ), the highest production comes from the states of Baja California, Jalisco and Michoacan (SIAP 2018). This last, is the main producer of blackberries $(96 \%$ of national production) and strawberries $(70 \%)$, while Jalisco is the principal producer of blueberries (46\%) and raspberry (71\%) (SIAP 2018).

Berry crops are attacked by a large number of plant pathogens including bacteria, fungi and oomycetes. These microorganisms induce diseases in most of the plant organs such as leaves, stems, inflorescences, fruits, and roots, reducing crop yields during pre- and postharvest. Several species are becoming a problem in berries worldwide, some as the bacteria Xanthomonas fragariae in strawberry (Kastelein et al. 2014; Kamangar et al. 2017; Wang et al. 2017), the oomycete Phytophthora cinnamomi inducing crown and root rot in V. corymbosum (Tamietti 2003; Larach et al. 2009; Huarhua et al. 2018), the fungi Fusarium oxysporum causing wilt disease in blackberry (Gordon et al. 2016; Pastrana et al. 2017) and blueberries (Liu et al. 2014; Moya-Elizondo et al. 2019), or several species of Lasiodiplodia in different berries, as Lasiodiplodia theobromae causing dieback in strawberry (Nam et al. 2016) or inducing stem blight and dieback of blueberry plants (Wright and Harmon 2009; Scarlett et al. 2019; Rodríguez-Gálvez et al. 2020).

Recently in Mexico, Neofusicoccum algeriense was detected in berries causing dieback in raspberry for the first time (Serret-López et al. 2017). In addition, Neopestalotiopsis rosae was reported inducing root rot, crown rot and leaf spot in strawberry in Central Mexico, causing losses of up to $50 \%$ of transplanted plants (Rebollar-Alviter et al. 2020). N. rosae is a new pathogen of strawberry that should be considered as an emerging threat to this fruit crop (Rebollar-Alviter et al. 2020).

The objective of the study was to compile all the references of diseases caused by bacteria, fungi and

To cite this paper: Garay-Serrano E, S Cruz-Esteban, SPF Pavia, GR Alvarado, N Gómez-Dorantes (2021). Pathogenic microorganisms infecting berries in Mexico. Intl J Agric Biol 25:1007-1015 


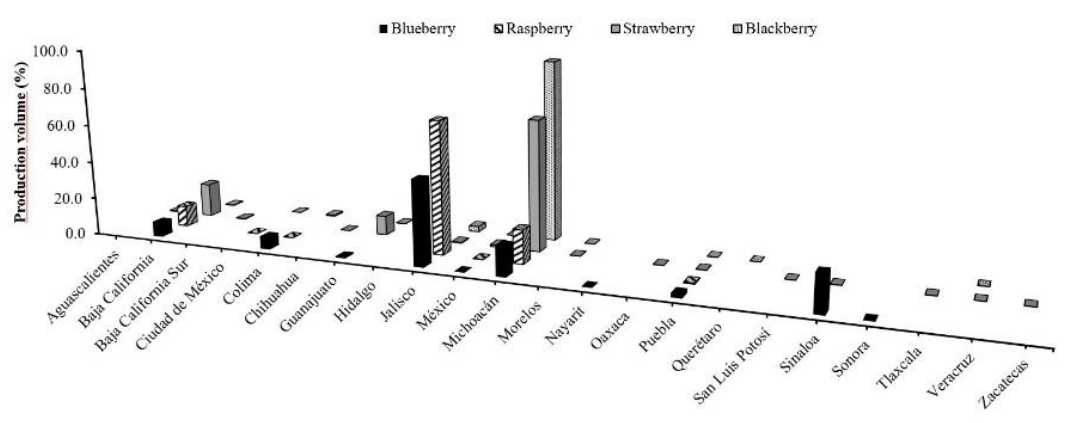

Fig. 1: Percentage of cultivated berries's production volume for each state in Mexico

oomycetes, on commercial crops and wild berries in Mexico. This work includes those reports where plant pathogens were identified using molecular and/or morphological techniques, and the studies were published in scientific papers, books or databases. We consider that this work will be useful for plant pathologists, plant breeders and other scientists that work with berries in Mexico and elsewhere.

\section{Materials and Methods}

We conducted a fully comprehensive search on databases of scientific articles, books, and conference proceedings containing reports of diseases of berries, including blackberry, blueberry, raspberry and strawberry, from Mexico during the last 64 years. Our interest was to focus on reports where molecular and/or morphological analyses were utilized to identify the pathogens affecting these crops. Additionally, a search was conducted in the National Fungus Collections of The Agricultural Research Service of the US Department of Agriculture (Farr and Rossman 2019).

The information collected was organized in a database that included hosts, locality, affected tissue of the plant, the name of the induced disease and the bibliographic reference. Subsequently, the information was ordered according to its taxonomic classification by kingdom, phylum, family, scientific name, and author of the species. The scientific names of the pathogens were corroborated using List of Prokaryotic names with Standing in Nomenclature (LPSN) (Parte et al. 2020; www.bacterio.net/-classifphyla.html.), Index Fungorum website (www.indexfungorum.org), and MycoBank Database (http://www.mycobank.org/).

The taxonomic names of the berry plants were verified using GBIF Secretariat (2019), also the distribution of berry plants reported was accessed to know if these plants have restricted or wide distribution in González-Elizondo and González-Elizondo (2014), and Rzedowski and Calderón (2005).

\section{Results}

Diseases of berries were reported in 17/32 states of Mexico. A total of the pathogens reported affecting berries crops in
Mexico is listed in Table 1.

\section{Pathogenic microorganisms by crop}

A total of 319 records of plant pathogens causing diseases on berries from Mexico during the period of 1956 to 2020, were detected in the accessed databases. These reports included diseases caused by bacteria, fungi, and oomycetes. The records included 54 genus of plant pathogens represented by 88 species. Five species corresponded to bacteria (5.7\%), 75 corresponded to fungi $(85.2 \%)$, and eight were oomycetes $(9.1 \%)$. Bacteria belong to 3 families, Stramenopila species appertain to 1 phylum (oomycota) and 2 families, and the fungi representing the largest pathogens group affecting berries, they are grouped in three phyla and 27 families.

Strawberry was the crop most affected with 53 pathogens, raspberry and blackberry presented 29 and 26 pathogens, respectively, while for blueberry there were only 16 pathogens reported (Table 2 ).

The genera of pathogen most reported were Agrobacterium, Alternaria, Botrytis, Colletotrichum, Fusarium, Gerwasia, Phytophthora, Podosphaera, Rhizoctonia and Verticillium. While the most frequently reported species were Agrobacterium radiobacter, Fusarium oxysporum, Gerwasia rubi, and Kuehneola loeseneriana affecting Rubus species; Botrytis cinerea, Peronospora sparsa in blackberry; Alternaria spp., Botrytis cinerea, Colletotrichum spp., Fusarium oxysporum, Ramularia grevilleana, Rhizoctonia solani, Verticillium albo-atrum and V. dahlia in strawberry; and Colletotrichum spp. and Thekopsora minima in blueberry.

\section{Botanical host species}

Most of the reports of berries diseases in Mexico were referred in cultivated species such as Vaccinium corymbosum (blueberry), Rubus idaeus (raspberry), Rubus ulmifolius (blackberry), and Fragaria x ananassa, a hybrid of strawberry. However, different botanical host species were also reported being affected by phytopathogens: the wild blackberries Rubus adenotrichos and Rubus humistratus, or the wild strawberry Fragaria mexicana and other species reported in Table 3 where the distributions are shown. 
Phytopathogenic Organisms Infecting Berries in Mexico / Intl J Agric Biol, Vol 25, No 5, 2021

Table 1: Phytopathogenic bacteria, oomycete and fungi inducing berries diseases reported in Mexico, arranged hierarchically by taxonomic group. Synonyms indicated in parentheses

\begin{tabular}{|c|c|c|c|c|c|c|}
\hline $\begin{array}{l}\text { PHYLLUM } \\
\text { BACTERIA }\end{array}$ & FAMILY & PATHOGEN & DISEASE & HOST & LOCALITY & REFERENCE \\
\hline \multirow[t]{2}{*}{ Proteobacteria } & Lysobacteraceae & Xanthomonas fragariae & Angular leaf spot & Strawberry & $\begin{array}{l}\text { Guanajuato, Michoacán, } \\
\text { Morelos }\end{array}$ & $\begin{array}{l}\text { García-Álvarez 1976; Narro-Sánchez et al. } \\
\text { 2006; Fernández-Pavía et al. } 2014\end{array}$ \\
\hline & Rhizobiaceae & $\begin{array}{l}\text { Agrobacterium } \\
\text { radiobacter } \\
\text { (=Agrobacterium } \\
\text { tumefaciens) }\end{array}$ & $\begin{array}{l}\text { Crown and cane } \\
\text { gall }\end{array}$ & $\begin{array}{l}\text { Raspberry, } \\
\text { strawberry, } \\
\text { blackberry }\end{array}$ & $\begin{array}{l}\text { Baja California, Ciudad de } \\
\text { México, Estado de } \\
\text { México, Michoacán }\end{array}$ & $\begin{array}{l}\text { García-Álvarez 1976, 1981, Fernández- } \\
\text { Pavía et al. 2015, López-Aranda et al. } 2016\end{array}$ \\
\hline \multirow[t]{3}{*}{ Tenericutes } & Acholeplasmataceae & $\begin{array}{l}\text { Candidatus Phytoplasma } \\
\text { spp. group 16SrXIII-(A/I)I }\end{array}$ & $\begin{array}{l}\text { Green petal, } \\
\text { periwinkle } \\
\text { virescence, inedible } \\
\text { fruit }\end{array}$ & $\begin{array}{l}\text { Blueberry, } \\
\text { raspberry, } \\
\text { strawberry, } \\
\text { blackberry }\end{array}$ & Jalisco, Michoacán & $\begin{array}{l}\text { Pérez-López and Dumonceaux 2016, Pérez- } \\
\text { López et al. } 2017\end{array}$ \\
\hline & & $\begin{array}{l}\text { Candidatus Phytoplasma } \\
\text { spp. group Aster yellow }\end{array}$ & $\begin{array}{l}\text { Green petal, } \\
\text { periwinkle plants } \\
\text { with virescence }\end{array}$ & Strawberry & Guanajuato, Michoacán & Narro-Sánchez et al. 2006 \\
\hline & & $\begin{array}{l}\text { Candidatus Phytoplasma } \\
\text { spp. group } 16 \text { SrXIII }\end{array}$ & $\begin{array}{l}\text { Green petal, } \\
\text { periwinkle plants } \\
\text { with virescence }\end{array}$ & Strawberry & Michoacán & Avendaño-Benequen et al. 2017 \\
\hline \multicolumn{7}{|c|}{ STRAMENOPILA } \\
\hline \multirow{7}{*}{ Oomycota } & & Phytophthora cactorum & $\begin{array}{l}\text { Leather rot on fruit } \\
\text { and crown rot and } \\
\text { wilt }\end{array}$ & Strawberry & $\begin{array}{l}\text { Estado de México, } \\
\text { Guanajuato, Michoacán }\end{array}$ & $\begin{array}{l}\text { Castro-Franco et al. 1993; Mendoza- } \\
\text { Zamora and Romero-Cova 1989; Narro- } \\
\text { Sánchez et al. 2006; Fernández-Pavía et al. } \\
2015\end{array}$ \\
\hline & & Phytophthora capsici & Fruit rot & Strawberry & Estado de México & Mendoza-Zamora and Romero-Cova 1989 \\
\hline & & $\begin{array}{l}\text { Phytophthora fragariae } \\
\text { var. fragariae }\end{array}$ & Red stele root rot & Strawberry & Michoacán & Ceja-Torres et al. 2008 \\
\hline & & $\begin{array}{l}\text { Phytophthora rubi } \\
\text { (=Phytophthora fragariae } \\
\text { var. rubi) }\end{array}$ & Root rot & Raspberry & $\begin{array}{l}\text { México, does not specify } \\
\text { locality }\end{array}$ & López-Aranda et al. 2016 \\
\hline & & Phytophthora spp. & $\begin{array}{l}\text { Root rot and dry } \\
\text { wilt }\end{array}$ & Strawberry & $\begin{array}{l}\text { Estado de México, } \\
\text { Guanajuato, Michoacán }\end{array}$ & $\begin{array}{l}\text { García-Álvarez 1976; Castro-Franco and } \\
\text { Dávalos 1990; Mendoza 1992; Fraire- } \\
\text { Cordero et al. 2003; Ceja-Torres et al. 2008, } \\
\text { Fernández-Pavía et al. } 2015\end{array}$ \\
\hline & Pythiaceae & Pythium aphanidermatum & Dry wilt & Strawberry & Michoacán & Ceja-Torres et al. 2008 \\
\hline & & Pythium spp. & Dry wilt & Raspberry & Jalisco & López-Aranda et al. 2016 \\
\hline \multirow{17}{*}{ Ascomycota } & Botryosphaeriaceae & Lasiodiplodia spp. & $\begin{array}{l}\text { Necrosis of leaves, } \\
\text { branches and stems }\end{array}$ & Blackberry & Michoacán & Contreras-Pérez et al. 2019 \\
\hline & & Neofusicoccum algeriense & Dieback & Raspberry & Michoacán & Serret-López et al. 2017 \\
\hline & & Neofusicoccum parvum & $\begin{array}{l}\text { Stem Blight and } \\
\text { Dieback }\end{array}$ & $\begin{array}{l}\text { Blueberry, } \\
\text { blackberry }\end{array}$ & Jalisco, Michoacán & Boyzo-Marin et al. 2016 \\
\hline & & Neofusicoccum spp. & Stem canker & Blueberry & Michoacán & Mondragón-Flores et al. 2012 \\
\hline & & Sphaeropsis spp. & Fruit rot & Strawberry & $\begin{array}{l}\text { Estado de México, } \\
\text { Guanajuato }\end{array}$ & García-Álvarez 1976 \\
\hline & Chaetomiaceae & Chaetomium spp. & Plant bud blight & Blueberry & Michoacán & Mondragón-Flores et al. 2012 \\
\hline & Cladosporiaceae & Cladosporium spp. & Blossom blight & Strawberry & México, Michoacán & Fraire-Cordero et al. 2003 \\
\hline & Diaporthaceae & $\begin{array}{l}\text { Phomopsis obscurans } \\
\text { (=Dendrophoma obscurans) }\end{array}$ & Leaf blight & Strawberry & Estado de México & Mendoza-Zamora and Romero-Cova 1988 \\
\hline & & Phomopsis spp. & Stem canker & Blueberry & Michoacán & Mondragón-Flores et al. 2012 \\
\hline & Didymellaceae & Ascochyta spp. & $\begin{array}{l}\text { Marginal leaf spot } \\
\text { or leaf blight }\end{array}$ & Strawberry & $\begin{array}{l}\text { Estado de México, } \\
\text { Guanajuato }\end{array}$ & Mendoza-Zamora and Romero-Cova 1988 \\
\hline & & Phoma spp. & $\begin{array}{l}\text { Stem canker, root } \\
\text { and crown rot }\end{array}$ & $\begin{array}{l}\text { Blueberry, } \\
\text { strawberry }\end{array}$ & $\begin{array}{l}\text { Estado de México, } \\
\text { Guanajuato, Michoacán }\end{array}$ & $\begin{array}{l}\text { Mendoza-Zamora and Romero-Cova 1989; } \\
\text { Mondragón-Flores et al. } 2012\end{array}$ \\
\hline & Dipodascaceae & Geotrichum spp. & Soft fruit rot & Strawberry & Michoacán & Fraire-Cordero et al. 2003 \\
\hline & Drepanopezizaceae & $\begin{array}{l}\text { Diplocarpon earlianum } \\
\text { (=Diplocarpon earliana) }\end{array}$ & Leaf scorch & Strawberry & $\begin{array}{l}\text { Estado de México, } \\
\text { Guanajuato, Morelos }\end{array}$ & $\begin{array}{l}\text { García-Álvarez 1976; Narro-Sánchez et al. } \\
\text { 2006; Fernández-Pavía et al. } 2015\end{array}$ \\
\hline & & $\begin{array}{l}\text { Diplocarpon fragariae } \\
\text { (=Marssonina fragariae) }\end{array}$ & Leaf scorch & Strawberry & Estado de México & Mendoza-Zamora and Romero-Cova 1988 \\
\hline & & $\begin{array}{l}\text { Marssonina } \text { spp. } \\
\text { (=Gloeosporium } \text { spp.) }\end{array}$ & Leaf scorch & Strawberry & $\begin{array}{l}\text { Aguascalientes, Estado de } \\
\text { México, Hidalgo, } \\
\text { Michoacán }\end{array}$ & $\begin{array}{l}\text { García-Álvarez 1981; Mendoza-Zamora and } \\
\text { Romero-Cova 1988; Fernández-Pavía et al. } \\
2015\end{array}$ \\
\hline & Elsinoaceae & Elsinoe veneta & Cane spot & $\begin{array}{l}\text { Raspberry, } \\
\text { blackberry }\end{array}$ & $\begin{array}{l}\text { Puebla, México, does not } \\
\text { specify locality }\end{array}$ & García-Álvarez 1976 \\
\hline & Erysiphaceae & $\begin{array}{l}\text { Podosphaera aphanis } \\
\text { (=Sphaerotheca macularis } \\
\text { f. fragariae) }\end{array}$ & Powdery mildew & Strawberry & $\begin{array}{l}\text { Estado de México, } \\
\text { Guanajuato, Michoacán }\end{array}$ & $\begin{array}{l}\text { García-Álvarez 1976; Mendoza-Zamora and } \\
\text { Romero-Cova 1988; Narro-Sánchez et al. } \\
2006\end{array}$ \\
\hline
\end{tabular}

Table 1: Continued 
Garay-Serrano et al. / Intl J Agric Biol, Vol 25, No 5, 2021

Table 1: Continued

\begin{tabular}{|c|c|c|c|c|c|}
\hline & $\begin{array}{l}\text { Podosphaera } \\
\text { macularis } \\
\text { (=Sphaerotheca } \\
\text { humuli) }\end{array}$ & Powdery mildew & Strawberry & $\begin{array}{l}\text { Chihuahua, Guanajuato, } \\
\text { Jalisco, Morelos }\end{array}$ & $\begin{array}{l}\text { García-Álvarez 1976; Fernández-Pavía } \\
\text { et al. } 2015\end{array}$ \\
\hline & Podosphaera spp. & Powdery mildew & Blackberry & Michoacán & $\begin{array}{l}\text { Tejera \& Ochoa 2004; Agustín and } \\
\text { García } 2007\end{array}$ \\
\hline \multirow[t]{3}{*}{ Glomerellaceae } & $\begin{array}{l}\text { Colletotrichum } \\
\text { acutatum }\end{array}$ & $\begin{array}{l}\text { Anthracnose fruit } \\
\text { rot }\end{array}$ & Strawberry & Michoacán & Espinoza-Altamirano et al. 2017 \\
\hline & $\begin{array}{l}\text { Colletotrichum } \\
\text { gloeosporioides }\end{array}$ & Anthracnose & Blackberry & $\begin{array}{l}\text { México, does not specify } \\
\text { locality }\end{array}$ & Horst 2013 \\
\hline & Colletotrichum spp. & $\begin{array}{l}\text { Anthracnose fruit } \\
\text { rot, leaf blight }\end{array}$ & $\begin{array}{l}\text { Blueberry, } \\
\text { raspberry, } \\
\text { strawberry }\end{array}$ & $\begin{array}{l}\text { Estado de México, } \\
\text { Guanajuato, Jalisco, } \\
\text { Michoacán, Morelos }\end{array}$ & $\begin{array}{l}\text { García-Álvarez 1976; Fraire-Cordero et } \\
\text { al. 2003; Narro-Sánchez et al. 2006; } \\
\text { Ceja-Torres et al. 2008; Mondragón- } \\
\text { Flores } \text { et al. 2012; Fernández-Pavía et } \\
\text { al. 2015; López-Aranda et al. } 2016\end{array}$ \\
\hline Meliolaceae & $\begin{array}{l}\text { Appendiculella } \\
\text { calostroma }\end{array}$ & Sooty mold & Blackberry & Veracruz & Farr and Rossman 2019 \\
\hline \multirow[t]{6}{*}{ Mycosphaerellaceae } & Cercospora spp. & Leaf Spot & $\begin{array}{l}\text { Raspberry, } \\
\text { strawberry }\end{array}$ & $\begin{array}{l}\text { Jalisco, México, does not } \\
\text { specify locality }\end{array}$ & $\begin{array}{l}\text { García- Álvarez 1981; Fernández-Pavía } \\
\text { et al. 2015; López-Aranda et al. } 2016\end{array}$ \\
\hline & Cercosporella spp. & Rosette & $\begin{array}{l}\text { Raspberry, } \\
\text { blackberry }\end{array}$ & Ciudad de México & García- Álvarez 1981 \\
\hline & $\begin{array}{l}\text { Mycosphaerella rubi } \\
\text { (=Septoria rubi) }\end{array}$ & Leaf spot & $\begin{array}{l}\text { Raspberry, } \\
\text { blackberry }\end{array}$ & $\begin{array}{l}\text { Puebla, México, does not } \\
\text { specify locality }\end{array}$ & $\begin{array}{l}\text { García- Álvarez 1976, 1981; Farr and } \\
\text { Rossman } 2019\end{array}$ \\
\hline & $\begin{array}{l}\text { Ramularia grevilleana } \\
\text { (=Ramularia tulasnei, } \\
\text { Mycosphaerella } \\
\text { fragariae })\end{array}$ & Leaf spot & Strawberry & $\begin{array}{l}\text { Estado de México, } \\
\text { Guanajuato, Michoacán, } \\
\text { México, does not specify } \\
\text { locality }\end{array}$ & $\begin{array}{l}\text { García- Álvarez 1976, 1981; Mendoza- } \\
\text { Zamora and Romero-Cova 1988; Narro- } \\
\text { Sánchez et al. 2006; Fernández-Pavía et } \\
\text { al. 2015; Farr and Rossman } 2019\end{array}$ \\
\hline & Septoria darrowii & Leaf spot & Blackberry & $\begin{array}{l}\text { México, does not specify } \\
\text { locality }\end{array}$ & Farr and Rossman 2019 \\
\hline & Septoria spp. & Leaf spot & $\begin{array}{l}\text { Raspberry, } \\
\text { blackberry }\end{array}$ & Ciudad de México & García- Álvarez 1976 \\
\hline \multirow[t]{13}{*}{ Nectriaceae } & $\begin{array}{l}\text { Albonectria } \\
\text { rigidiuscula } \\
\text { (=Fusarium } \\
\text { rigidiusculum })\end{array}$ & Root and crown rot & Strawberry & Estado de México & $\begin{array}{l}\text { Mendoza-Zamora and Romero-Cova } \\
1989\end{array}$ \\
\hline & Cylindrocarpon spp. & Black Root Rot & Strawberry & Guanajuato, Michoacán & $\begin{array}{l}\text { Ceja-Torres et al. 2008; Mariscal- } \\
\text { Amaro et al. } 2017\end{array}$ \\
\hline & $\begin{array}{l}\text { Fusarium } \\
\text { brachygibbosum }\end{array}$ & Dry wilt & Strawberry & Guanajuato & Mariscal-Amaro et al. 2017 \\
\hline & Fusarium equiseti & Dry wilt & Raspberry & Jalisco & López-Aranda et al. 2016 \\
\hline & $\begin{array}{l}\text { Fusarium fujikuroi } \\
\text { (=Fusarium } \\
\text { subglutinans, } F . \\
\text { verticillioides, } F \text {. } \\
\text { moniliforme })\end{array}$ & Dry wilt & $\begin{array}{l}\text { Raspberry, } \\
\text { strawberry }\end{array}$ & $\begin{array}{l}\text { Jalisco, Guanajuato, } \\
\text { Michoacán }\end{array}$ & $\begin{array}{l}\text { Mendoza-Zamora and Romero-Cova } \\
\text { 1989; López-Aranda et al. 2016; } \\
\text { Mariscal-Amaro et al. } 2017\end{array}$ \\
\hline & Fusarium oxysporum & Dry wilt & $\begin{array}{l}\text { Blackberry, } \\
\text { raspberry, } \\
\text { strawberry }\end{array}$ & $\begin{array}{l}\text { Estado de México, } \\
\text { Guanajuato, Jalisco, } \\
\text { Michoacán, Morelos }\end{array}$ & $\begin{array}{l}\text { Téliz-Ortíz et al. 1986; Mendoza- } \\
\text { Zamora and Romero-Cova 1989; Castro- } \\
\text { Franco and Dávalos 1990; Ceja-Torres } \\
\text { et al. 2008; Gordon et al. 2016; López- } \\
\text { Aranda et al. 2016; Mariscal-Amaro et } \\
\text { al. 2017; Bárcenas-Santana et al. } 2019\end{array}$ \\
\hline & Fusarium oxysporum & Dry wilt & $\begin{array}{l}\text { Blackberry, } \\
\text { raspberry, } \\
\text { strawberry }\end{array}$ & $\begin{array}{l}\text { Estado de México, } \\
\text { Guanajuato, Jalisco, } \\
\text { Michoacán, Morelos }\end{array}$ & $\begin{array}{l}\text { Téliz-Ortíz et al. 1986; Mendoza- } \\
\text { Zamora and Romero-Cova 1989; Castro- } \\
\text { Franco and Dávalos 1990; Ceja-Torres } \\
\text { et al. 2008; Gordon et al. 2016; López- } \\
\text { Aranda et al. 2016; Mariscal-Amaro et } \\
\text { al. 2017; Bárcenas-Santana et al. } 2019\end{array}$ \\
\hline & $\begin{array}{l}\text { Fusarium oxysporum } \\
\text { f. spp. mori }\end{array}$ & Dry wilt & Blackberry & $\begin{array}{l}\text { Michoacan, México, does } \\
\text { not specify locality }\end{array}$ & $\begin{array}{l}\text { Pastrana et al. 2017; Hernández-Cruz et } \\
\text { al. } 2020\end{array}$ \\
\hline & $\begin{array}{l}\text { Fusarium oxysporum } \\
\text { f. spp. fragariae }\end{array}$ & $\begin{array}{l}\text { Fusarium wilt of } \\
\text { strawberry }\end{array}$ & Strawberry & Guanajuato, Michoacán & $\begin{array}{l}\text { Dávalos-González et al. 2004; Narro- } \\
\text { Sánchez et al. } 2006\end{array}$ \\
\hline & Fusarium proliferatum & Vascular Wilt & Strawberry & Guanajuato & Mariscal-Amaro et al. 2017 \\
\hline & $\begin{array}{l}\text { Fusarium } \\
\text { pseudonygamai }\end{array}$ & Dry wilt & Strawberry & Guanajuato & Mariscal-Amaro et al. 2017 \\
\hline & Fusarium roseum & Dry wilt & Strawberry & Estado de México & $\begin{array}{l}\text { Mendoza-Zamora and Romero-Cova } \\
1989\end{array}$ \\
\hline & Fusarium spp. & Dry wilt & $\begin{array}{l}\text { Raspberry, } \\
\text { strawberry, } \\
\text { blackberry }\end{array}$ & $\begin{array}{l}\text { Ciudad de México, Estado } \\
\text { de México, Guanajuato, } \\
\text { Michoacán, México, does } \\
\text { not specify locality }\end{array}$ & $\begin{array}{l}\text { García-Álvarez 1976, 1981; Mendoza- } \\
\text { Zamora and Romero-Cova 1989; Castro- } \\
\text { Franco and Dávalos 1990; Mendoza } \\
\text { 1992; Dávalos-González et al. 2004; } \\
\text { Fernández-Pavía et al. } 2015\end{array}$ \\
\hline
\end{tabular}


Phytopathogenic Organisms Infecting Berries in Mexico / Intl J Agric Biol, Vol 25, No 5, 2021

Table 1: Continued

\begin{tabular}{|c|c|c|c|c|c|}
\hline & $\begin{array}{l}\text { Neocosmospora solani } \\
(=\text { Fusarium solani })\end{array}$ & Crown and root rot & Raspberry, strawberry & $\begin{array}{l}\text { Estado de México, } \\
\text { Guanajuato, Jalisco, } \\
\text { Michoacán }\end{array}$ & $\begin{array}{l}\text { Mendoza-Zamora and Romero-Cova } \\
\text { 1989; Ceja-Torres et al. 2008; López- } \\
\text { Aranda et al. 2016; Mariscal-Amaro } \text { et } \\
\text { al. } 2017\end{array}$ \\
\hline \multirow[t]{2}{*}{ Pestalotiopsidaceae } & Neopestalotiopsis rosae & Crown and root rot & Strawberry & Michoacán & Rebollar-Alviter et al. 2020 \\
\hline & Pestalotiopsis spp. & Crown and root rot & Blueberry, strawberry & Michoacán, Puebla & $\begin{array}{l}\text { Fraire-Cordero et al. } 2003 \text {; } \\
\text { Mondragón-Flores } \text { et al. 2012; } \\
\text { Morales-Mora et al. } 2019\end{array}$ \\
\hline Phyllostictaceae & Phyllosticta spp. & $\begin{array}{l}\text { Phyllosticta leaf } \\
\text { spot }\end{array}$ & Blueberry, strawberry & $\begin{array}{l}\text { Baja California, } \\
\text { Estado de México, } \\
\text { Michoacán, }\end{array}$ & $\begin{array}{l}\text { García-Álvarez 1976; Mendoza- } \\
\text { Zamora and Romero-Cova 1988; } \\
\text { Mondragón-Flores et al. } 2012\end{array}$ \\
\hline \multirow[t]{3}{*}{ Plectosphaerellaceae } & Verticillium albo-atrum & Verticillium wilt & Raspberry, strawberry & $\begin{array}{l}\text { Guanajuato, Jalisco, } \\
\text { Michoacán, México, } \\
\text { does not specify } \\
\text { locality. }\end{array}$ & $\begin{array}{l}\text { García-Álvarez 1976, 1981; Ceja- } \\
\text { Torres } \text { et al. 2008; Fernández-Pavía et } \\
\text { al. 2015; López-Aranda et al. } 2016\end{array}$ \\
\hline & Verticillium dahliae & Verticillium wilt & Strawberry & $\begin{array}{l}\text { Estado de México, } \\
\text { Guanajuato, } \\
\text { Michoacán }\end{array}$ & $\begin{array}{l}\text { Téliz-Ortíz et al. 1986; Castro-Franco } \\
\text { and Dávalos 1990; Mendoza 1992; }\end{array}$ \\
\hline & Verticillium spp. & Verticillium wilt & Raspberry, strawberry & $\begin{array}{l}\text { Guanajuato, } \\
\text { Michoacán, México, } \\
\text { does not specify } \\
\text { locality }\end{array}$ & $\begin{array}{l}\text { Narro-Sánchez et al. 2006; López- } \\
\text { Aranda et al. } 2016\end{array}$ \\
\hline \multirow[t]{5}{*}{ Pleosporaceae } & Alternaria alternata & Leaf Spot & Strawberry & Guanajuato & Mariscal-Amaro et al. 2017 \\
\hline & Alternaria spp. & $\begin{array}{l}\text { Leaf spot, dry wilt, } \\
\text { soft fruit rot, root } \\
\text { rot }\end{array}$ & $\begin{array}{l}\text { Blueberry, raspberry, } \\
\text { strawberry, blackberry }\end{array}$ & $\begin{array}{l}\text { Aguascalientes, Baja } \\
\text { California, Durango, } \\
\text { Estado de México, } \\
\text { Guanajuato, Jalisco, } \\
\text { Michoacán, Oaxaca }\end{array}$ & $\begin{array}{l}\text { García-Álvarez 1976, 1981; Fraire- } \\
\text { Cordero et al. 2003; Narro-Sánchez et } \\
\text { al. 2006; Mondragón et al. 2012; } \\
\text { Fernández-Pavía et al. 2015; López- } \\
\text { Aranda et al. } 2016\end{array}$ \\
\hline & Bipolaris spp. & Blueberry blight & Blueberry & Michoacán & Mondragón et al. 2012 \\
\hline & Curvularia spp. & Stem scab & Blueberry & Michoacán & Mondragón et al. 2012 \\
\hline & Stemphylium spp. & Silver stain & Blueberry & Michoacán & Mondragón et al. 2012 \\
\hline \multirow[t]{2}{*}{ Sclerotiniaceae } & Botrytis cinerea & $\begin{array}{l}\text { Gray mold, } \\
\text { Botrytis blossom } \\
\text { blight }\end{array}$ & Strawberry, blackberry & $\begin{array}{l}\text { Colima, Estado de } \\
\text { México, Guanajuato, } \\
\text { Jalisco, México, } \\
\text { does not specify } \\
\text { locality, Michoacán, } \\
\text { Morelos }\end{array}$ & $\begin{array}{l}\text { García-Álvarez 1976, 1981; Mendoza- } \\
\text { Zamora and Romero-Cova 1988; } \\
\text { Tejera and Ochoa 2004; Narro- } \\
\text { Sánchez et al. 2006; Agustín and } \\
\text { García 2007; Fernández-Pavía et al. } \\
\text { 2015; Álvarez-Medina et al. } 2017\end{array}$ \\
\hline & Botrytis spp. & Gray mold & Strawberry & Michoacán & Fraire-Cordero et al. 2003 \\
\hline Venturiaceae & Coleroa chaetomium & Leaf spot & Blackberry & Estado de México & Romero-Cova 1990 \\
\hline Xylariaceae & Rosellinia spp. & Root rot & Raspberry, blackberry & Durango & García-Álvarez 1976 \\
\hline \multirow[t]{3}{*}{ Ceratobasidiaceae } & Rhizoctonia fragariae & Root rot & Strawberry & Michoacán & Ceja-Torres et al. 2008 \\
\hline & Rhizoctonia solani & Root rot, fruit rot & Raspberry, strawberry & $\begin{array}{l}\text { Estado de México, } \\
\text { Guanajuato, Jalisco, } \\
\text { México, does not } \\
\text { specify locality, } \\
\text { Michoacán, Morelos, } \\
\text { Nayarit, Sonora }\end{array}$ & $\begin{array}{l}\text { García-Álvarez 1976, 1981; Téliz- } \\
\text { Ortíz et al. 1986; Mendoza-Zamora } \\
\text { and Romero-Cova 1989; Castro-Franco } \\
\text { and Dávalos 1990; Mendoza 1992; } \\
\text { López-Aranda et al. } 2016\end{array}$ \\
\hline & Rhizoctonia spp. & Root rot & Strawberry & $\begin{array}{l}\text { Guanajuato, } \\
\text { Michoacán }\end{array}$ & Narro-Sánchez et al. 2006 \\
\hline \multirow[t]{7}{*}{ Phragmidiaceae } & Gerwasia pittieriana & Rust & Raspberry, blackberry & $\begin{array}{l}\text { Morelos, México, } \\
\text { does not specify } \\
\text { locality }\end{array}$ & León-Gallegos and Cummnins 1981 \\
\hline & Gerwasia rubi & Rust & Raspberry, blackberry & $\begin{array}{l}\text { Michoacán, Morelos, } \\
\text { Veracruz, México, } \\
\text { does not specify } \\
\text { locality, }\end{array}$ & $\begin{array}{l}\text { López et al. 1980; León-Gallegos and } \\
\text { Cummnins 1981; López } 1984\end{array}$ \\
\hline & Gymnoconia nitens & Rust & Raspberry, blackberry & $\begin{array}{l}\text { Puebla, México, } \\
\text { does not specify } \\
\text { locality }\end{array}$ & León-Gallegos and Cummnins 1981 \\
\hline & Kuehneola arthurii & Rust & Blackberry & $\begin{array}{l}\text { México, does not } \\
\text { specify locality }\end{array}$ & León-Gallegos and Cummnins 1981 \\
\hline & Kuehneola loeseneriana & Rust & Raspberry, blackberry & $\begin{array}{l}\text { Veracruz, México, } \\
\text { does not specify } \\
\text { locality, }\end{array}$ & $\begin{array}{l}\text { López et al. 1980; León-Gallegos and } \\
\text { Cummnins 1981; López 1984; } \\
\text { Hernández and Hennen } 2003\end{array}$ \\
\hline & $\begin{array}{l}\text { Mainsia rubi } \\
(=\text { Uromyces rubi) }\end{array}$ & Rust & Raspberry, blackberry & $\begin{array}{l}\text { México, does not } \\
\text { specify locality, } \\
\text { Puebla, Veracruz }\end{array}$ & $\begin{array}{l}\text { Watson 1971; García-Álvarez 1976; } \\
\text { Farr and Rossman } 2019\end{array}$ \\
\hline & $\begin{array}{l}\text { Phragmidium } \\
\text { mexicanum (=Frommea } \\
\text { mexicana) }\end{array}$ & Rust & Strawberry & $\begin{array}{l}\text { México, does not } \\
\text { specify locality }\end{array}$ & $\begin{array}{l}\text { Cummins and Stevenson 1956; } \\
\text { Watson 1971; García-Álvarez 1976; } \\
\text { Fernández-Pavía et al. } 2015\end{array}$ \\
\hline
\end{tabular}

Table 1: Continued 
Table 1: Continued

\begin{tabular}{|c|c|c|c|c|c|c|}
\hline & & Phragmidium occidentale & Rust & Raspberry & Veracruz & Carrion and Galvan 1987 \\
\hline & & Phragmidium spp. & Rust & Raspberry, blackberry & Puebla & García-Álvarez 1981 \\
\hline & Pucciniastraceae & $\begin{array}{l}\text { Naohidemyces vaccinii } \\
\text { (=Pucciniastrum vaccinii, } \\
\text { Naohidemyces vacciniorum) }\end{array}$ & Late leaf rust & Blueberry & $\begin{array}{l}\text { Durango, México, } \\
\text { does not specify } \\
\text { locality }\end{array}$ & León-Gallegos and Cummnins 1981 \\
\hline & & Pucciniastrum americanum & Leaf rust & Raspberry & $\begin{array}{l}\text { Estado de México, } \\
\text { Jalisco, Michoacán }\end{array}$ & $\begin{array}{l}\text { Rebollar-Alviter et al. 2001a, b; } \\
\text { Marín et al. } 2019\end{array}$ \\
\hline & & Pucciniastrum spp. & Leaf rust & Blueberry & Michoacán & Mondragón-Flores et al. 2012 \\
\hline & & Thekopsora minima & Leaf rust & Blueberry & $\begin{array}{l}\text { Jalisco, Michoacán, } \\
\text { México, does not } \\
\text { specify locality }\end{array}$ & $\begin{array}{l}\text { Rebollar-Alviter et al. 2011; Horst } \\
2013\end{array}$ \\
\hline Mucoromycota & Mucoraceae & Mucor spp. & Soft fruit rot & Strawberry & Michoacán & Fraire-Cordero et al. 2003 \\
\hline & Rhizopodaceae & $\begin{array}{l}\text { Rhizopus stolonifer (=Rhizopus } \\
\text { nigricans) }\end{array}$ & Soft fruit rot & Strawberry, blackberry & $\begin{array}{l}\text { Estado de México, } \\
\text { Guanajuato, México, } \\
\text { does not specify } \\
\text { locality, Michoacán }\end{array}$ & $\begin{array}{l}\text { García-Álvarez 1976; Fraire-Cordero } \\
\text { et al. 2003; Chávez-Díaz et al. 2014; } \\
\text { Fernández-Pavía et al. } 2015\end{array}$ \\
\hline
\end{tabular}

Table 2: Species of pathogenic microorganisms reported affecting berries, organized by Kingdom

\begin{tabular}{lllll}
\hline & Bacteria & Stramenopila & Fungi & Pathogenic species by crop \\
\hline Blue berry & 1 & 0 & 15 & 16 \\
Rasp berry & 2 & 2 & 25 & 29 \\
Straw berry & 5 & 5 & 43 & 53 \\
Black berry & 2 & 1 & 23 & 26 \\
Total pathogenic species by group & 5 & 8 & 75 & 88 \\
\hline
\end{tabular}

Table 3: Berry plant species reported to be affected for plant pathogens microorganism in Mexico

\begin{tabular}{|c|c|c|c|}
\hline Family & Berry & Scientific name & Characteristics and distribution \\
\hline \multirow[t]{2}{*}{ Ericaceae } & Blueberry & Vaccinium corymbosum $\mathrm{L}$. & Widely cultivated species. E.U. and North Hemisphere* \\
\hline & & Vaccinium geminiflorum Kunth & Mexican blueberry. Dwarf blueberry, Distributed from Alaska to Guatemala** \\
\hline \multirow[t]{2}{*}{ Rosaceae } & Raspberry & Rubus idaeus L. & Widely cultivated species. Native of Europe and North Asia* \\
\hline & & Rubus trilobus Moc. \& Sessé ex Ser. & Guatemala and southern and central Mexico* \\
\hline \multirow[t]{2}{*}{ Rosaceae } & Strawberry & $\begin{array}{l}\text { Fragaria } x \text { ananassa } \\
\text { (Weston) Duchesne }\end{array}$ & North America to Patagonia. \\
\hline & & Fragaria mexicana Schltdl. & Wild Mexican strawberry. Mexico* \\
\hline \multirow[t]{4}{*}{ Rosaceae } & Blackberry & Rubus adenotrichos Schltdl. & $\begin{array}{l}\text { Wild blackberry. Distributed from central Mexico to Ecuador. The fruits are highly collected in } \\
\text { Michoacan*** }\end{array}$ \\
\hline & & Rubus fruticosus $\mathrm{L}$. & Cultivated species. North and south Hemisphere* \\
\hline & & Rubus humistratus Steud & Wild blackberry. Endemic species of Mexico*** \\
\hline & & Rubus ulmifolius Schott & Cultivated species. Europe, North Africa, America and Oceania** \\
\hline
\end{tabular}

\section{Discussion}

Berries plant protection include several strategies, but one of them is to know the pathogens that potentially affect the crops, especially considering that pest (understood as pathogens, animals, and weed) in global level, could cause annual crop yield losses from 20 to $40 \%$ of the production (Oerke 2006; Savary et al. 2012).

In this compilation of phytopathogens affecting berries in Mexico, fungi were the largest group reported with 75 of a total of 88 species of microorganism. The most common fungal pathogens included Botrytis cinerea, Fusarium oxysporum and Colletotrichum spp.; while the bacterium Agrobacterium rhizobacter, and the oomycete Peronospora sparsa and Phytophthora cactorum, were some of the most common pathogens reported. These fungi and bacteria are considered amongst the top plant pathogens due to the severe economic losses that cause worldwide, and their scientific importance (Dean et al. 2012; Mansfield et al. 2012). Other reported pathogens such as Thekopsora minima in blueberry (Rebollar-Alviter et al. 2011), and Rhizoctonia solani in strawberry (Mouden et al. 2016; Amrutha and Vijayaraghavan 2019; Errifi et al. 2019) should also be considered as highly important due to the reductions on yields that cause (Ahmed et al. 2017).

A major deficiency in $35 \%$ of the reports that were analyzed was the lack of identification to the species level. More recent studies have included molecular techniques to assess the taxonomic species (Boyzo-Marin et al. 2016; Serret-López et al. 2017; Contreras-Pérez et al. 2019). However, in the cases of Colletotrichum and Fusarium, the presence of species complexes makes difficult to establish the correct identification of the involved species due to the probable presence of cryptic species. To identify species in these species complexes is necessary to use several molecular markers (Pastrana et al. 2017; Hernández-Cruz et 
al. 2020). The use of multilocus identification may detect new species such as Neopestalotiopsis rosae reported in strawberry (Rebollar-Alviter et al. 2020).

Although pathogens in wild species of berries in Mexico have been scarcely studied, some wild hosts were referenced (Table 3). These plants might be capable of harboring pathogens that could serve as inoculum source reservoirs for the infection of nearby crops (Grünwald et al. 2001; Pak et al. 2017).

The information of phytopathogens affecting berries in Mexico will allow to researchers to detect areas of opportunity to focus on, and for technicians to have a benchmark about all the pathogens in each berry crop that have been reported. Knowing the pathogens will help to plant management strategies to combat infection caused by phytopathogens.

\section{Conclusion}

Records of bacteria, oomycetes and pathogenic fungi reported in berries in Mexico since 1956 to 2020 added up to a total of 88 species. Phytopathogenic bacteria are represented by $5.7 \%$ of the species, $9.1 \%$ correspond to oomycetes and $85.2 \%$ to fungi. Still there are phytopathogenic species that need more in-depth taxonomic studies, coupled with this, there are several lineages integrating species complex that need to delve into molecular, genetic and physiological studies to understand in a better way the strategies to control the field diseases.

\section{Acknowledgements}

EG thanks to Jazmín Cortés Martínez for the support provided in the collection of initial data.

\section{Author Contributions}

EG and SC generated the idea of the study. EG, SC, SPF, and NG collected the data. EG prepared the initial manuscript; performed the tables. SC, SPF, GR analyzed the data to be considered. All authors commented on the manuscript, reviewed drafts of the paper, and approved the final draft.

\section{Conflicts of Interest}

All other authors declare no conflicts of interest

\section{Data Availability}

Not applicable.

\section{Ethics Approval}

Not applicable.

\section{References}

Agustín JA, DLS García (2007). La Producción Agropecuaria y Forestal de la Región de Cotija Los Reyes, Mich. Centro Regional Universitario UACH, Morelia Michocan, México

Ahmed RE, HH Mohmed, E Embaby, A Attia (2017). Studies on growth and yield losses in strawberry and its relation to soil borne diseases in major producing governorates in Egypt. Egypt Acad J Biol Sci 8:2130

Álvarez-Medina A, HV Silva-Rojas, SG Leyva-Mir, N Marbán-Mendoza, A Rebollar-Alviter (2017). Resistencia de Botrytis cinerea de fresa (Fragaria x ananassa Duch.) a fungicidas en Michoacán, Mexico. Agrociencia 51:783-798

Amrutha P, R Vijayaraghavan (2019). Characterization of Rhizoctonia solani causing fruit rot of strawberry (Fragaria $\mathrm{x}$ ananassa Duch.) in wayanad and in vitro evaluation of fungicides, organic preparations and bioagents for its management. J Hortic Sci 14:155-160

Avendaño-Benequen M, HV Silva-Rojas, N Marbán-Mendoza, A RebollarAlviter(2017). Mexican periwinkle virescence phytoplasma associated with phyllody and virescence in strawberry (Fragaria $\mathrm{x}$ ananassa Duch.) in Michoacán, Mexico. Eur J Plant Pathol 147:451-454

Bárcenas-Santana D, D Guillén-Sánchez, C Yazmín-Basaldua, MD de Lorena Ramos-García, M Valle-de la Paz (2019). Etiología de la secadera de la fresa (Fragaria spp.) en Morelos, México. Rev Mex Fitopatol 37:1-10

Boyzo-Marin J, A Rebollar-Alviter, HR Silva-Rojas, G RamirezMaldonaldo (2016). First report of Neofusicoccum parvum causing stem blight and dieback of blueberry in Mexico. Plant Dis 100:2524-2524

Carrion G, M Galvan (1987). Plant pathogens fungi from the state of Veracruz, Uredinales V. Rev Mex Mic 3:149-159

Castro-Franco J, , P Dávalos (1990). Etiología de "la secadera" o pudrición de la raíz y corona de la fresa en Irapuato, Guanajuato. Rev Mex Fitopatol 8:80-86

Castro-Franco J, PA Dávalos-González, E Redondo-Juárez (1993). El agente causal de la pudrición "coriácea" de la fruta de la fresa en el Bajío. Rev Mex Fitopatol 11:103-106

Ceja-Torres LF, G Mora-Aguilera, D Téliz, A Mora-Aguilera, P SánchezGarcía, C Muñoz-Ruiz, B Tlapal-Bolaños, RDL Torre-Almaráz (2008). Ocurrencia de hongos y etiología de la secadera de la fresa con diferentes sistemas de manejo agronómico. Agrociencia 42:451-461

Chávez-Díaz IF, V Angoa-Pérez, S López-Díaz, MG Velázquez-del Valle, AN Hernández-Lauzardo (2014). Antagonistic bacteria with potential for biocontrol on Rhizopus stolonifer obtained from blackberry fruits. Fruits 69:41-46

Contreras-Pérez M, G Santoyo-Pizano, S de los Santos Villalobos, MA Gutiérrez-García, MC Orozco-Mosqueda, MC Rocha-Granados (2019). First report of Lasiodiplodia on blackberry plants (Rubus subgenus Eubatus) in the Michoacan state, Mexico. Rev Mex Fitopatol 37:479-485

Cummins GB, JA Stevenson (1956). A check list of North American rust fungi (Uredinales). In: A checklist of north American rust fungi, pp:193

Dávalos-González PA, AE Jofre-Garfias, AR Hernández-Razo, J NarroSánchez, J Castro-Franco, N Vázquez-Sánchez, R Bujanos-Muñiz (2004). Strawberry breeding for the Central Plateau of Mexico. Acta Hortic 708:547-552

Dean R, JALV Kan, ZA Pretorius, KE Hammond-Kosack, AD Pietro, PD Spanu, JJ Rudd, M Dickman, R Kahmann, J Ellis, GD Foster (2012). The top 10 fungal pathogens in molecular plant pathology. Mol Plant Pathol 13:414-430

Errifi A, AO Touhami, N Mouden, AM El Batnan, N El Hazzat, K Selmaoui, R Benkirane, A Douira (2019). Pathogenicity of Rhizoctonia solani towards distinct varieties of cultivated strawberry plants in Morocco. Plant Cell Biotechnol Mol Biol 20:1086-1099

Espinoza-Altamirano D, HV Silva-Rojas, SG Leyva-Mir, N MarbánMendoza, A Rebollar-Alviter (2017). Sensitivity of Colletotrichum acutatum isolates obtained from strawberry to tiophanate-methyl and azoxystrobin fungicides. Rev Mex Fitopatol 35:186-203 
FAOSTAT R (2020). FAOSTAT database. Food and Agriculture Organization of the United Nations. Retrieved 02/08/2020 Available at: http://www.fao.org/faostat/es/\#data (Accessed: 02 August 2020)

Farr DF, AY Rossman (2019). Fungal Databases, U.S. National Fungus Collections, ARS, USDA. Retrieved in July 2019, Available at: https://nt.ars-grin.gov/fungaldatabases/specimens/specimens.cfm). (Accessed: 16 July 2019)

Fernández-Pavía SP, R Gregorio-Cipriano, G Rodríguez-Alvarado, YL Fernández-Pavía, A Mondragón-Flores, N Gómez-Dorantes, HL Saldaña, RR Fernández, J Herrera-Camacho (2015). Enfermedades de Especies Vegetales en Mexico, $1^{\text {st }}$ Edition. Universidad Michoacana de San Nicolás de Hidalgo. Morevalladolid, Morelia, Michoacán, México

Fernández-Pavía SP, G Rodríguez-Alvarado, E Garay-Serrano, R CárdenasNavarro (2014).First report of Xanthomonas fragariae causing angular leaf spot on strawberry plants in Mexico. Plant Dis 98:682-682

Fraire-Cordero ML, MJ Yañez-Morales, DN Angel, GV Galvez (2003). Hongos patógenos en frutos de fresa (Fragaria $\mathrm{x}$ ananassa Duch.) en postcosecha. Rev Mex Fitopatol 21:285-291

García-Álvarez M (1981). Enfermedades de Plantas en la República Mexicana, pp:93. Limusa. D.F. Mexico

García-Álvarez M (1976). Primer catálogo de enfermedades de plantas Mexicanas. Fitofilo 71:45-168

GBIF Secretariat (2019). GBIF Backbone Taxonomy. Checklist dataset Available at: https://doi.org/10.15468/39omei accessed via GBIF.org (Accessed: 11 June 2020)

González-Elizondo MS, M González-Elizondo (2014). Ericaceae. In: Flora del Bajjio y de Regiones Adyacentes, Vol. 183, pp:1-123

Gordon TR, SC Kirkpatrick, PM Henry, M Kong, JC Broome (2016). First report of a wilt disease of blackberry caused by Fusarium oxysporum in California. Plant Dis 100:1018-1018

Grünwald NJ, WG Flier, AK Sturbaum, E Garay-Serrano, TBMV Bosch, CD Smart, JM Matuszak, H Lozoya-Saldaña, LJ Turkensteen, WE Fry (2001). Population structure of Phytophthora infestans within the Toluca valley region of central Mexico. Phytopathology 91:882-890

Hernández JR, JF Hennen (2003). Rust fungi causing galls, witches' brooms, and other abnormal plant growths in northwestern Argentina. Mycologia 95:728-755

Hernández-Cruz A, A Saldivia-Tejeda, HV Silva-Rojas, D Fuentes-Aragón, C Nava-Díaz, L Martínez-Bolaños, A Rebollar-Alviter (2020) Evaluation of full-season programs for the management of Fusarium wilt of blackberry caused by a new lineage of the Fusarium oxysporum species complex. Crop Prot 134:1-11

Horst RK (2013). Field Manual of Diseases on Fruits and Vegetables, p:139. Springer Netherlands

Huarhua M, J Flores, R Acuña, W Apaza (2018). Morphological and molecular identification of Phytophthora cinnamomi Rands as causal agent of crown and root rot in Blueberry (Vaccinium corymbosum) in Peru. Peruv J Agron 2:14-21

Kamangar SB, JV Vaerenbergh, S Kamangar, M Maes (2017). First report of angular leaf spot on strawberry caused by Xanthomonas fragariae in Iran. Plant Dis 101:1031

Kastelein P, M Krijger, R Czajkowski, VDPS Zouwen, VDR Schoor, H Jalink, VDJM Wolf (2014). Development of Xanthomonas fragariae populations and disease progression in strawberry plants after sprayinoculation of leaves. Plant Pathol 63:255-263

Larach A, X Besoain, E Salgado (2009). Crown and root rot of highbush blueberry caused by Phytophthora cinnamomi and P. citrophthora and cultivar sensitivity. $J$ Agric Nat Res 36:433-442

León-Gallegos ML, GB Cummnins (1981). Uredinales (royas) de Mexico, Vol. 2, p:492. Instituto Nacional de Investigaciones Agrícolas, Culiacán, Sinaloa, México

Liu YH, T Lin, CS Ye, CQ Zhang (2014). First report of Fusarium wilt in blueberry (Vaccinium corymbosum) caused by Fusarium oxysporum in China. Plant Dis 98:1158-1158

López A (1984). The rust fungi of Mexico, Vol. 22, pp:158-163. Reports of the Tottori Mycological Institute, Japan

López A, G Carrión, M Galván, D Martínez (1980). Hongos Fitopatógenos del estado de Veracruz, I. Uredinales. Bol Soc Mex Mic 14:81-100
López-Aranda JM, F Gomez, M Puga, R Zamora, O Daugovish, MA Cotero (2016). Chemical soil fumigation for raspberry nursery in Jalisco (Mexico). J Berry Res 6:37-46

Mansfield J, S Genin, S Magori, V Citovsky, M Sriariyanum, P Ronald, M Dow, V Verdier, SV Beer, MA Machado, I Toth, G Salmond, GD Foster (2012). Top 10 plant pathogenic bacteria in molecular plant pathology. Mol Plant Pathol 13:614-629

Marín Cortez FA, W Chan-Cupul, MTB Nava, HA Hernández-Ortega, G Manzo-Sánchez, EG Velasco (2019). Biological control of late leaf rust disease [Pucciniastrum americanum (Farl.) Arthur] in raspberry (Rubus idaeus L.) using two biological products: Bacillus subtilis (Fungizard®) and Larrea tridentata botanic extract (CleanCrop®) under screenhouse conditions. Idesia 37:125-133

Mariscal-Amaro LA, A Rivera-Yerena, PA Dávalos-González, D ÁvilaMartínez (2017). Situación actual de hongos asociados a la secadera de la fresa (Fragaria $\times$ ananassa Duch.) en Guanajuato, México. Agrociencia 51:673-681

Mendoza ZC (1992). Enfermedades fungosas de hortalizas y fresa. In: Manejo Fitosanitario de las Hortalizas en México, pp:273-288. Centro de Entomología y Acarología. Chapingo, México

Mendoza-Zamora C, S Romero-Cova (1989). Enfermedades de la fresa Fragaria chiloensis var. ananassa en Villa Guerrero, Edo. México II: Identificación e incidencia de los hongos que atacan los frutos. Rev Mex Fitopatol 7:7-19

Mendoza-Zamora C, S Romero-Cova (1988). Enfermedades de la fresa Fragaria chiloensis var. ananassa en Villa Guerrero, Edo. de México. I: Identificación e incidencia de los hongos que atacan el follaje. Rev Mex Fitopatol 6:120-130

Mondragón-Flores A, J López-Medina, S Ochoa-Ascencio, M GutiérrezContreras (2012). Hongos asociados a la parte aérea del arándano en Los Reyes, Michoacán, Mexico. Rev Mex Fitopatol 30:141-144

Morales-Mora LA, SJ Martínez-Salgado, MAVD Ita, MP Andrade-Hoyos, HV Silva-Rojas, O Romero-Arenas (2019). First report of leaf spot and anthracnosis caused by Pestalotiopsis spp. on strawberry in Puebla, México. Plant Dis 103:2668-2671

Mouden N, R Benkirane, AO Touhami, A Douira (2016) Fungal species associated with collapsed strawberry plants cultivated in strawberries plantations in Morocco. Intl J Curr Res 8:29108-29117

Moya-Elizondo EA, H Doussoulin, JS Martin, B Ruiz, PD Valle (2019). First report of Fusarium oxysporum causing Fusarium wilt on blueberry (Vaccinium corymbosum) in Chile. Plant Dis 103:2669-2669

Nam MH, MS Park, HS Kim, TI Kim, EM Lee, JD Park, HG Kim (2016) First report of dieback caused by Lasiodiplodia theobromae in strawberry plants in Korea. Mycobiology 44:319-324

Narro-Sánchez J, PA Dávalos-González, R Velasquez-Valle, J CastroFranco (2006). Main strawberry diseases in Irapuato, Guanajuato and Zamora, Michoacan, Mexico. Acta Hortic 708:167-172

Oerke EC (2006). Crop losses to pests. J Agric Sci 144:31-43

Pak D, MP You, V Lanoiselet, MJ Barbetti (2017). Reservoir of cultivated rice pathogens in wild rice in Australia. Eur J Plant Pathol 147:295311

Parte AC, JS Carbasse, JP Meier-Kolthoff, LC Reimer, M Göker (2020) List of Prokaryotic names with Standing in Nomenclature (LPSN) moves to the DSMZ. Intl J Syst Evol Microbiol 70:5607-5612

Pastrana AM, SC Kirkpatrick, M Kong, JC Broome, TR Gordon (2017). Fusarium oxysporum f. spp. mori, a new forma specialis causing Fusarium wilt of blackberry. Plant Dis 101:2066-2072

Pérez-López E, TJ Dumonceaux (2016). Detection and identification of the heterogeneous novel subgroup $16 \mathrm{SrXIII-(A/I)} \mathrm{I} \mathrm{phytoplasma}$ associated with strawberry green petal disease and Mexican periwinkle virescence. Intl J Syst Evol Microbiol 66:4406-4415

Pérez-López E, D Rodríguez-Martínez, CY Olivier, M Luna-Rodríguez, TJ Dumonceaux (2017). Molecular diagnostic assays based on cpn60 UT sequences reveal the geographic distribution of subgroup 16SrXIII-(A/I) I phytoplasma in Mexico. Sci Rep 7:1-14

Rebollar-Alviter A, HV Silva-Rojas, D Fuentes-Aragón, U AcostaHernández, M Martínez-Ruiz, BE Parra-Robles (2020). An emerging strawberry fungal disease associated with root rot, crown rot and leaf spot caused by Neopestalotiopsis rosae in Mexico. Plant Dis 104:2054-2059 
Rebollar-Alviter A, AM Minnis, LJ Dixon, LA Castlebury, MR RamírezMendoza, HV Silva-Rojas, G Valdovinos-Ponce (2011). First report of leaf rust of blueberry caused by Thekopsora minima in México. Plant Dis 95:772-772

Rebollar-Alviter A, HV Silva-Rojas, LXZ Molina, MA Ellis (2001a). Identificación y biología del agente causal de la roya de la frambuesa roja (Rubus idaeus L.) en México. Rev Mex Fitopatol 19:168-174

Rebollar-Alviter A, SG Leyva-Mir, G Mora-Aguilera, G Valdovinos Ponce, S Romero-Cova (2001b). Identificación y biología del agente causal de la roya de la frambuesa roja (Rubus idaeus L.) en México. Rev Mex Fitopatol 19:168-174

Rodríguez-Díaz KJ, HV Silva-Rojas, J Boyzo-Marin, SD Segura-Ledesma, SG Leyva-Mir, A Rebollar-Alviter (2017). Molecular detection of Peronospora sparsa in sources of primary inoculum and components of resistance in wild blackberry species. Eur J Plant Pathol 149:845851

Rodríguez-Gálvez E, S Hilário, A Lopes, A Alves (2020). Diversity and pathogenicity of Lasiodiplodia and Neopestalotiopsis species associated with stem blight and dieback of blueberry plants in Peru. Eur J Plant Pathol 157:89-102

Romero-Cova S (1990). Identificación de algunos hongos fitopatógenos nuevos o poco conocidos en México. Rev Mex Fitopatol 8:1-8

Rzedowski J, G Calderón (2005). Rosaceae. Flora del Bajio y de Regiones Adyacentes, Vol. 135, pp: 1-157

SADER-SIAP (2019). Servicio de Información Agroalimentaria y Pesquera. Secretaría de Agricultura y Desarrollo Rural. Panorama agroalimentario (2019). https://nube.siap.gob.mx/gobmx_publicaciones_siap/

Savary S, A Ficke, JN Aubertot, C Hollier (2012). Crop losses due to diseases and their implications for global food production losses and food security. Food Sec 4:519-537
Scarlett KA, LA Shuttleworth, D Collins, CT Rothwell, DI Guest, R Daniel (2019). Botryosphaeriales associated with stem blight and dieback of blueberry (Vaccinium spp.) in New South Wales and Western Australia. Aust Plant Pathol 48:45-57

Serret-López RE, B Tlapal-Bolaños, SG Leyva-Mir, KC Correia, M Camacho-Tapia, F Méndez-Jaimes, JM Tovar-Pedraza (2017). First report of Neofusicoccum algeriense causing dieback of red raspberry in Mexico. Plant Dis 101:1673-1673

SIAP (2018). Servicio de Información Agroalimentaria y Pesquera. Estadística de la Producción Agrícola de 2018. Available at: http://infosiap.siap.gob.mx/gobmx/datosAbiertos_a.php, (Accessed: 28 August 2019)

Tamietti G (2003). First report of Phytophthora cinnamomi on high-bush blueberry in Italy. Plant Dis 87:451-451

Tejera H, F Ochoa (2004). La zarzamora ante los retos productivos, del mercado y del desarrollo local. Universidad Autónoma Chapingo, p:175. México

Téliz-Ortíz D, A Mendoza-Herrera, J Sandoval (1986). Enfermedades de la fresa en México. Rev Mex Fitopatol 4:1-12

Wang J, HL Wei, RK Chang, HQ Liu, YH Wang (2017). First report of strawberry bacterial angular leaf spot caused by Xanthomonas fragariae in Tianjin, China. Plant Dis 101:19491949

Watson AJ (1971). Foreign bacterial and fungus diseases of food, forage, and fiber crops: An annotated list (No. 418). VII, p: 111. Agricultural Research Service, US Dept. of Agriculture, Washington DC, USA

Wright AF, PF Harmon (2009). First report of Lasiodiplodia theobromae associated with stem blight of southern highbush blueberries in Florida. Plant Dis 93:962-962 